\title{
Colegios visibles: \\ estructuras de coparticipación en tribunales de tesis doctorales de biblioteconomía y documentación en España
}

\section{Por Carlos Olmeda-Gómez, Antonio Perianes-Rodríguez, Maa Antonia Ovalle-Perandones y Félix de Moya-Anegón}

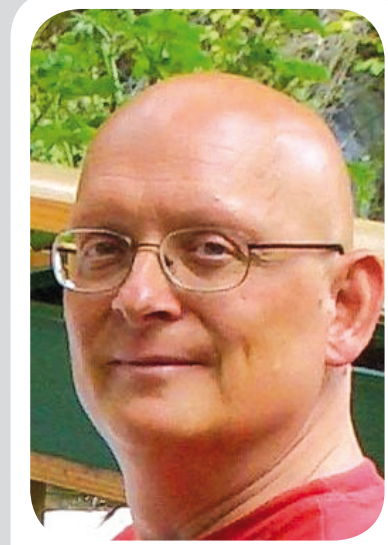

Carlos Olmeda-Gómez es profesor titular en el Departamento de Biblioteconomía y Documentación de la Universidad Carlos III de Madrid e investigador del grupo $\mathrm{SCl}$ mago.

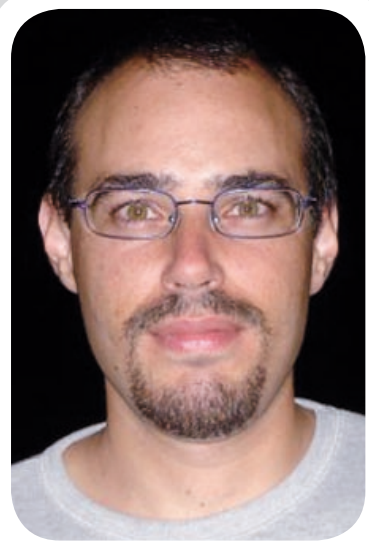

Antonio Perianes-Rodríguez es profesor ayudante en el Departamento de Biblioteconomía y Documentación de la Universidad Carlos III de Madrid e investigador del grupo SClmago.

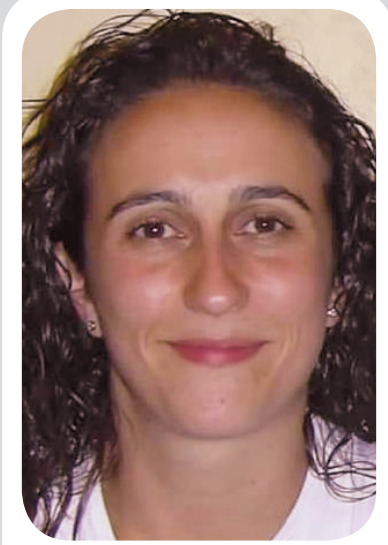

Ma Antonia Ovalle-Perandones es profesora en el Departamento de Biblioteconomía y Documentación de la Universidad Carlos III de Madrid e investigadora del grupo SClmago.

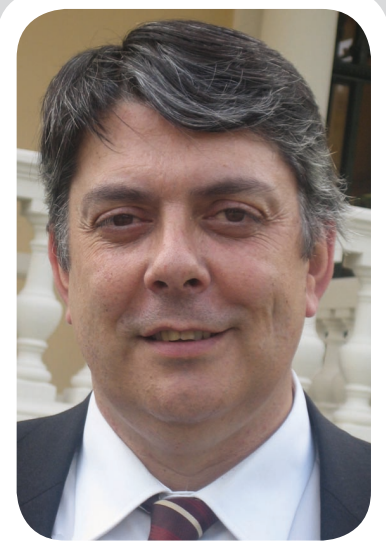

Félix de Moya-Anegón es profesor de Investigación en el Instituto de Políticas y Bienes Públicos y director del grupo SCImago.

Resumen: Se presentan los resultados de un análisis de la red social de coparticipación de profesores en tribunales de tesis doctorales de biblioteconomía y documentación entre 1993 y 2006. Se emplean dos mecanismos para generar subredes basados en reducción por grado y k-core y se aplica un análisis de cluster jerárquico, según el método de Ward. La red resultante identifica cinco clases integradas por aquellos actores que ocupan posiciones sociales similares, al mismo tiempo que se argumentan los mecanismos relevantes desde el punto de vista interpretativo, basados en la similitud y la proximidad física.

Palabras clave: Redes científicas académicas, Análisis de redes sociales, Análisis de conglomerados, Biblioteconomía y documentación, España

Title: Visible colleges: co-membership structure of Spanish PhD juries in Library and Information Science

Abstract: The results of a study of social networking by professors' co-memberships in library and information science PhD juries between 1993 and 2006 are presented. Degree reduction and $k$-core methods are used to extract sub-networks and a Ward's hierarchical cluster analysis is performed. The resulting network identifies five classes, whose members are actors occupying similar social positions, and at the same time different mechanisms, based mainly in similarity and physical proximity, are proposed from the interpretative point of view.

Keywords: Academic scientific networks, Social network analysis, Cluster analysis, Library and information science, Spain

Olmeda-Gómez, Carlos; Perianes-Rodríguez, Antonio; Ovalle-Perandones, Ma Antonia; Moya-Anegón, Félix de. "Colegios visibles: estructuras de coparticipación en tribunales de tesis doctorales de biblioteconomía y documentación en España”. En: El profesional de la información, 2009, enero-febrero, v. 18, n. 1, pp. 41-49.

DOI: 10.3145/epi.2009.ene.06

\section{Introducción}

La ciencia académica es un trabajo profesional, un modo de producción de conocimientos cuyas reglas sociales son inseparables de las pautas que delimitan su campo de conocimiento. En todas las profesiones hay grupos de colegas que tratan de autorregular el desarrollo profesional mediante numerosas tareas de control. Una de las más conocidas en la sociedad académica es la validación de los aspirantes a formar parte de una 
disciplina, basándose en su poder para confirmar el grado de experto que posee un aspirante. Las disciplinas y las estructuras burocráticas que las soportan, es decir los departamentos universitarios, son los encargados de la formación y certificación de los nuevos científicos y la obtención del grado de doctor sanciona el tránsito desde la posición de estudiante a la posición de estar legitimado para entrar en el mercado académico. Otras formas conocidas de control en el seno del ejercicio profesional tienen que ver con la distribución de recompensas materiales, la distribución del reconocimiento científico y el control sobre el desarrollo y promoción de las carreras profesionales.

\section{"La configuración social e intelectual de las disciplinas es una preocupación permanente en la sociología de la ciencia y en la Information Science"}

Desde la sociología de la ciencia, se sugieren diferentes perspectivas teóricas que explican cómo se estructuran las disciplinas. Entre las posibles, hay que destacar aquella que subraya la importancia de los procesos de comunicación y de socialización a partir de la teoría de redes y la generación de círculos sociales (Crane, 1975). Según este enfoque, los mayores centros de investigación son creadores de escuelas de pensamiento o tradiciones investigadoras (López Yepes, 2002) que se difunden a través de colegios invisibles (Price; Beaver, 1966), redes de comunicación o exportando profesionales hacia otros centros que integran el mercado académico de la disciplina (Valle Gastaminza, 2002).

La configuración social e intelectual de las disciplinas es una preocupación permanente en la sociología de la ciencia y en la Information science. En esta última disciplina, los análisis de redes de citas (Small; Crane, 1979) y de co-citas han producido detalladas representaciones, tanto de las interrelaciones de las especialidades en un determinado dominio geográfico (Vargas-Quesada et al., 2008), como de las personas que las integran (Small, 1980; White; McCain, 1998), descubriendo las leyes que gobiernan el crecimiento y topología de las redes, como en el caso de las de coautoría de trabajos científicos (Newman, 2004). Desde esta doble perspectiva se puede reconocer la importancia que tiene el concepto de "redes" para múltiples propósitos de análisis sobre la organización intelectual y social de las disciplinas, que va más allá de este enunciado, ya que, como afirma Ziman (2003, p. 132-133), "las teorías científicas se pueden representar como redes abstractas en las que los nodos de hechos y/o conceptos están entrelaza- dos en muchas dimensiones mediante leyes, fórmulas, semejanzas de familias u otras relaciones funcionales".

El trabajo profesional de los científicos no se reduce a la comunicación mediante procesos formales basados en las publicaciones científicas que se conservan de forma permanente. También se ejerce empleando canales informales dirigidos a audiencias restringidas, como son las asistencias y debates en congresos y reuniones de asociaciones profesionales, tribunales, discusiones con colaboradores, circulación de borradores, conversaciones informales en reuniones varias, de carácter más efímero (Garvey; Griffith, 1964), cuyas posibilidades se han visto incrementadas y diversificadas desde las pasadas dos décadas, con el correo electrónico, los grupos de discusión, la World Wide Web e internet (Barjak, 2006).

En los procesos informales también es posible detectar la red de relaciones en las que quedan inmersas las prácticas sociales de los científicos de cualquier disciplina, y pueden ser analizadas desde el punto de vista estructural con vistas a la detección de comunidades, colegios invisibles, grupos informales o grupos que comparten valores y/o paradigmas (Brieger, 1976). De acuerdo con Freeman (1984, p. 203), "una disciplina científica emergente o consolidada puede caracterizarse por los densos y frecuentes vínculos entre sus profesionales $\mathrm{y}$, en realidad, la presencia de esas densas relaciones, puede usarse para delimitar las fronteras de una disciplina científica”.

\section{"Los vínculos intelectuales y sociales no pueden ordenarse siempre de forma precisa (White, 2004)"}

El "colegio visible" en una disciplina estaría integrado por aquellos grupos que legitiman ante una audiencia la validez de las contribuciones de carácter científico y participan en los procesos de certificación, reclutamiento, promoción y distribución de recompensas, basándose en criterios de reputación, prestigio, mérito o autoridad intelectual. Pueden citarse como ejemplos los miembros de tribunales de tesis doctorales; de contrataciones o exámenes públicos, de consejos de redacción de revistas científicas o de comités de programas científicos de congresos y reuniones profesionales, dirigentes de asociaciones profesionales, evaluadores, cargos académicos, etc.

Esta investigación se ha diseñado con el fin de examinar la red social y de interacciones profesionales que ha tenido lugar entre los profesores universitarios de biblioteconomía y documentación (ByD) de las universidades españolas, a partir de los datos de copar- 
ticipación en tribunales de tesis doctorales. Se revisa brevemente la literatura previa publicada al respecto y se presenta la metodología empleada. A continuación, se presentan los resultados del análisis de redes llevado a cabo, se discuten los resultados y se presentan unas breves conclusiones.

\section{Antecedentes}

Las tesis doctorales españolas en ByD han sido objeto de diferentes trabajos en los que se abordan los datos de producción, temáticas, centros de lectura, directores o aspectos relacionados con la legislación (López Yepes, 2001; Fuentes; González, 2002; Zapico et al., 2002; Orera-Orera, 2004; López Yepes et al., 2005).

No son frecuentes los trabajos que emplean el análisis de redes. Sierra (2003) lo utiliza para estudiar los tribunales de ciencias físicas y físico matemáticas para plazas de profesor de investigación en el Consejo Superior de Investigaciones Científicas, entre 1985 y 2002. Para ello genera una matriz rectangular de modo dos, en la que las columnas son los tribunales y las filas, los miembros pertenecientes a los diferentes tribunales y comprueba la existencia de un reducido núcleo de investigadores con una alta tasa de participación en tribunales. Perianes-Rodríguez (2004), sobre la base de las tesis leídas en los departamentos de documentación de Granada y de la Universidad Carlos III entre 1993 y 2003, genera una matriz de adyacencia, simétrica y cuadrada, en la que tanto las columnas como las filas son las universidades a las que pertenecen los miembros asistentes a los tribunales de los respectivos departamentos. De las redes no dirigidas obtenidas, calcula posteriormente valores de centralización y de cohesión y las representa de forma gráfica. Delgado et al. (2006) realizan una investigación a partir de los datos de 213 tesis de bibliometría extraídas de la base de datos $T e$ seo. Analizan las características más sobresalientes de la producción y efectúan un análisis de redes a partir de tres matrices: de coparticipación en tribunales, de tribunales por director y de participación por universidad. Calculan valores de centralización y de cohesión de los principales coparticipantes en los tribunales y caracterizan a los actores más prominentes en escuelas en las que se puede clasificar la bibliometría.

\section{Metodología}

Para el análisis de la red social entre profesores de ByD, se aplica el método de análisis de redes sociales (Scott, 1992), partiendo de la hipótesis de que los datos utilizados son sólo indicadores de vínculos sociales existentes entre los miembros. No es pues, un análisis basado en criterios bibliométricos, sino en datos sociométricos. Si se quieren conocer las diversas estructuras subyacentes en una disciplina, se precisan múltiples grafos para describirla puesto que "los vínculos intelectuales y sociales no pueden ordenarse siempre de forma precisa" (White et al., 2004, p. 112).

\subsection{Recopilación de información}

Los datos se han extraído a partir de las tesis doctorales en el área de $\mathrm{ByD}$, leídas en departamentos o facultades que imparten esta titulación en las universidades españolas entre 1993 y 2006 . Han sido obtenidos en junio de 2008 de la base de datos Teseo, gestionada en la actualidad por el Ministerio de Ciencia e Innovación, cuyas ventajas y limitaciones ya son conocidas (Delgado et al., 2006, p. 497).

\section{https://www.micinn.es/teseo/login.jsp}

Los datos inexactos se han completado con información sobre tesis doctorales accesibles en las webs de las universidades analizadas que disponen de ella. La estrategia de búsqueda se ha basado en la combinación de la información que figura en los campos respectivos en la interfaz de interrogación de la base. Se han empleado también como argumento, los nombres de directores detectados en trabajos previos (Zapico et al., 2002, p. 713). Se han descartado de la investigación los trabajos leídos en unidades administrativas motivo de análisis pero con una orientación distinta a las del área analizada (tesis de arte leídas en departamentos de ByD) y aquellas que, aun siendo del área por su temática, se han leído en departamentos distintos de los establecidos a priori (tesis de biblioteconomía leídas en departamentos de ingeniería). El resultado ha sido la obtención una base de datos con 177 documentos, gestionada con $\mathrm{Mi}$ crosoft Access 2007 para su tratamiento y explotación.

\subsection{Análisis de datos}

Se ha construido una matriz de adyacencia cuadrada sobre la coparticipación de 311 profesores que han formado parte de 177 tribunales. En esa matriz cada columna y cada fila representan un profesor, o vértice de la red y la celda de intersección suma valores en función de las coincidencias en tribunales con otro profesor. La celda está vacía cuando no se coincide con otro miembro. Como la coincidencia entre dos profesores es recíproca, los vínculos que los unen no son dirigidos. Los vértices de la diagonal se representan con ceros ya que ningún profesor coincide consigo mismo.

El tipo de análisis en el que estamos interesados busca, en primer lugar, 1) detectar las regularidades en

"Los nodos integrantes en cada clase ocupan posiciones sociales similares en la red, lo que no implica que sean subgrupos cohesionados" 
la estructura de conjunto; 2) dado el tamaño de la red inicial, nos interesa usar alguna aproximación estadística que nos pueda identificar subredes más pequeñas que puedan posteriormente ser analizadas usando métodos más sofisticados, y 3)una vez delimitada una subred núcleo, estudiar los patrones de enlace para identificar y agrupar los nodos cuyas posiciones son más similares, basándonos en las relaciones que mantienen con el resto de los actores.

- Se han calculado los siguientes indicadores: número de enlaces, densidad, grado promedio, diámetro, centralización de grado y centralización de intermediación.

Se entiende por "densidad", la relación que hay entre el número de enlaces presentes y el número de enlaces posibles de la red. La densidad de la red no es un indicador muy útil, ya que depende del tamaño de la misma. Es mejor observar el número de vínculos que tiene cada uno de los vértices, es decir, el grado de los vértices. Los vértices con mayor grado son los que se encontrarán en las partes densas de la red.

El "promedio de grado" de todos los vértices es una medida de cohesión estructural de la red, con la ventaja de que su valor es independiente del tamaño de la red y hace posible la comparación entre redes de diferentes tamaños.

El "diámetro" es la longitud de la mayor distancia geodésica entre cualquier par de nodos.

La "centralización de grado" es un índice que mide la variación de grado de los vértices o nodos dividido por la variación máxima de grado posible en una red del mismo tamaño. Por intermediación de un nodo se entiende la proporción de distancias geodésicas en la red que incluyen ese nodo.

La "centralización de la intermediación" mide la variación de los valores de intermediación de los vértices dividido por la variación máxima posible de los valores de intermediación de una red del mismo tamaño (Wasserman; Faust, 1994).

- Cuando sea posible, las representaciones de redes no deben estar abarrotadas de enlaces y de nodos. Para podarlas existen diferentes métodos, de los que en este trabajo se emplean dos. El primero se ha utilizado en la tarea de preproceso de la red inicial. Ha consistido en reducir de forma recursiva el número de nodos de la red con un grado menor de 6. Para el análisis posterior de la red se ha elegido el método de $k$-core. Un subconjunto de vértices se denomina $k$-core si cada vértice del subconjunto está conectado al menos a $k$ vértices del mismo subconjunto (Batagelj; Zaversnik, 2003).

- Para generar clusters de nodos que compartan patrones similares de enlaces con los demás, se ha se- guido el siguiente procedimiento indirecto (Batagelj et al., 1992): a partir de la matriz resultante del análisis $k$ core, se ha construido otra de disimilaridades, a la que se ha aplicado un análisis de cluster jerárquico aglomerativo basado en el método de Ward. Los nodos de la red que se obtienen han sido distribuidos espacialmente empleando el algoritmo Kamada Kwai. La representación final se obtiene a partir de un fichero gráfico SVG (Moya et al., 2006, p. 263).

\section{Resultados}

Los datos descriptivos se presentan en las tablas 1, 2 y 3. En las universidades Carlos III de Madrid, Granada y Complutense, se ha defendido el $74 \%$ de las tesis doctorales de acuerdo con las condiciones del análisis. Les siguen la Universidad de Murcia, Salamanca, Barcelona, Politécnica de Valencia, Zaragoza, Extremadura y Alcalá de Henares.

\begin{tabular}{|l|c|c|}
\hline \multicolumn{1}{|c|}{ Universidad } & No tesis & \% tesis \\
\hline UC3M & 46 & 25,9 \\
\hline UGR & 43 & 24,9 \\
\hline UCM & 42 & 23,7 \\
\hline UM & 22 & 12,4 \\
\hline USAL & 9 & 5,0 \\
\hline UB & 7 & 3,9 \\
\hline UPV & 3 & 1,6 \\
\hline Unizar & 2 & 1,1 \\
\hline UEX & 2 & 1,1 \\
\hline UAH & 1 & 0,5 \\
\hline Total & $\mathbf{1 7 7}$ & $\mathbf{1 0 0 , 0}$ \\
\hline
\end{tabular}

Tabla 1. Distribución de tesis de ByD por institución. 1993-2006

En el período estudiado los directores más productivos han sido: Moya-Anegón (UGR), López-Yepes y Sagredo-Fernández (UCM), Moreiro-González y Sanz-Casado $(U C 3 M)$ y Rodríguez-Muñoz (UM), cuya dirección conjunta acumula el 48,8\% del total de tesis leídas. ¿Es una concentración elevada? Price (1980) señaló que el núcleo de autores del colegio invisible podría ser estimado en "aproximadamente, la raíz cuadrada del número total de científicos autores", que concentrarían la mitad de la producción. En este caso, en el colegio visible de $\mathrm{ByD}$, la clase más productiva es, aproximadamente, la mitad de la raíz cuadrada del número total de directores de tesis.

La coparticipación en tribunales, tanto de la propia universidad de adscripción como en otras universidades, se encuentra consignada de forma parcial en 
la tabla 3, ya que no se presentan los datos de los 295 profesores restantes que han intervenido al menos en un tribunal. En este colegio visible destacan Moreiro-
González y Caridad-Sebastián $(U C 3 M)$ ya que son los actores más destacados en esta red sociocognitiva de la ByD española. El 50\% de la clase con más la-

\begin{tabular}{|c|c|c|c|c|c|c|}
\hline Rango & Director & $\begin{array}{l}\text { Tesis } \\
\text { dirigidas }\end{array}$ & $\begin{array}{c}\% \\
\text { tesis }\end{array}$ & $\begin{array}{c}\% \\
\Delta\end{array}$ & $\begin{array}{c}\text { Dirigidas } \\
\text { fuera }\end{array}$ & $\%$ \\
\hline 1 & Moya-Anegón, Félix (UGR) & 24 & 13,5 & 13,5 & 1 & 4,17 \\
\hline 2 & López-Yepes, José (UCM) & 21 & 11,8 & 25,3 & - & - \\
\hline 3 & Sagredo-Fernández, Félix (UCM) & 12 & 6,7 & 32,0 & 1 & 8,33 \\
\hline 4 & Moreiro-González, José Antonio (UC3M) & 10 & 5,6 & 37,6 & - & - \\
\hline 5 & Rodríguez-Muñoz, José-Vicente (UM) & 10 & 5,6 & 43,2 & - & - \\
\hline 6 & Sanz-Casado, Elías (UC3M) & 10 & 5,6 & 48,8 & 1 & 10,00 \\
\hline 7 & Caridad-Sebastián, Mercedes (UC3M) & 6 & 3,3 & 52,1 & 1 & 16,60 \\
\hline 8 & Jiménez-Contreras, Evaristo (UGR) & 6 & 3,3 & 55,4 & 1 & 16,60 \\
\hline 9 & Pinto-Molina, María (UGR) & 6 & 3,3 & 58,7 & 3 & 50,00 \\
\hline 10 & Ros-García, Juan (UM) & 6 & 3,3 & 62,0 & 4 & 66,60 \\
\hline 11 & Ayuso-García, Dolores (UM) & 5 & 2,8 & 64,8 & 1 & 20,00 \\
\hline 12 & Frías, José-Antonio (USAL) & 5 & 2,8 & 67,6 & - & - \\
\hline 13 & Ruiz-Rodríguez, Antonio (UGR) & 5 & 2,8 & 70,4 & - & - \\
\hline 14 & Ramos-Simón, L.-Fernando (UCM) & 4 & 2,2 & 72,6 & 2 & 50,00 \\
\hline 15 & Espinosa-Temiño, Blanca (UCM) & 4 & 2,2 & 74,8 & - & - \\
\hline
\end{tabular}

Tabla 2. Directores de 4 o más tesis de ByD. 1993-2006

\begin{tabular}{|c|c|c|c|c|c|}
\hline Rango & Nombre & $\begin{array}{c}\mathrm{N}^{\circ} \mathrm{de} \\
\text { tribunales }\end{array}$ & $\%$ & $\begin{array}{c}N^{\circ} \text { de } \\
\text { tribunales } \\
\text { de fuera }\end{array}$ & $\%$ \\
\hline 1 & Moreiro-González, José Antonio (UC3M) & 45 & 25,4 & 25 & 55,50 \\
\hline 2 & Caridad-Sebastián, Mercedes (UC3M) & 36 & 20,3 & 7 & 19,44 \\
\hline 3 & Sagredo-Fernández, Félix (UCM) & 27 & 15,2 & 10 & 37,04 \\
\hline 4 & Valle-Gastaminza, Félix del (UCM) & 22 & 12,4 & 8 & 36,36 \\
\hline 5 & Sanz-Casado, Elías (UC3M) & 19 & 10,7 & 9 & 47,30 \\
\hline 6 & López-Yepes, José (UCM) & 17 & 9,6 & 11 & 64,71 \\
\hline 7 & Pinto-Molina, María (UGR) & 16 & 9,0 & 14 & 87,50 \\
\hline 8 & Jiménez-Contreras, Evaristo (UGR) & 14 & 7,9 & 4 & 30,70 \\
\hline 9 & Moscoso-Castro, Purificación $(U A H)$ & 12 & 6,7 & 12 & 100,00 \\
\hline 10 & Frías, José-Antonio (USAL) & 12 & 6,7 & 10 & 83,33 \\
\hline 11 & Ros-García, Juan (UM) & 11 & 6,2 & 11 & 100,00 \\
\hline 12 & Martínez-Montalvo, Esperanza $(U A H)$ & 10 & 5,6 & 10 & 100,00 \\
\hline 13 & Hernández-Pérez, Tony(UC3M) & 10 & 5,6 & 2 & 20,00 \\
\hline 14 & López-Yepes, Alfonso (UCM) & 10 & 5,6 & 6 & 60,00 \\
\hline 14 & Moya-Anegón, Félix de (UGR) & 10 & 5,6 & 6 & 60,00 \\
\hline 16 & Rodríguez-Muñoz, José-Vicente (UM) & 10 & 5,6 & 3 & 60,00 \\
\hline
\end{tabular}


zos sociales proceden de las universidades de Madrid, y nueve de los primeros dieciséis son profesores catedráticos.

La red de coparticipación es una red no dirigida, no es ponderada y no tiene ciclos dirigidos. Los valores de la red completa y de las dos subredes, se presentan en la tabla 4. A medida que se aplican las reducciones, se incrementa la densidad, crecen los valores normalizados de grado, disminuye el diámetro y disminuyen el número de nodos y de enlaces. Así se logra una subred de cincuenta y cinco nodos que posibilita su análisis para conocer la colección de actores que son similares en sus vínculos con los demás.

El dendograma de la figura 1 representa la solución de cluster completa. Aquí, la ubicación de los profesores del núcleo refleja la totalidad del conjunto de relaciones en la matriz de disimilaridad. La raíz une los dos principales clusters, uno integrado por trece miembros y otro por cuarenta y dos. El cluster más pequeño de profesores (13), es más similar internamente $(81,82)$ que el cluster más grande $(55)(82,26)$.

El método elegido mezcla vértices en clusters, y los más pequeños los vuelve a mezclar en uno de tamaño superior. El dendograma ayuda a identificar el número de clusters en la partición. Así, hemos generado cinco clases equivalentes de vértices basadas en cluster jerárquico, que dibujamos en la figura 2, asignando un color que los identifica visualmente y etiquetándolo con las siglas de la universidad a la que pertenece el catedrático de mayor antigüedad. En dorado el cluster $U C 3 M 1$, en rojo el cluster $U C 3 M 2$, en azul el cluster $U C M 1$, con verde el cluster $U C M 2$, y en violeta

\begin{tabular}{|l|r|r|r|}
\hline \multicolumn{1}{|c|}{ Medidas } & \multicolumn{1}{|c|}{$\begin{array}{c}\text { Red } \\
\text { completa }\end{array}$} & $\begin{array}{c}\text { Red } \\
\text { podada } \\
\text { grado } \\
\mathbf{6} \mathbf{6}\end{array}$ & $\begin{array}{c}\text { Red } \boldsymbol{k} \text { - } \\
\text { core }\end{array}$ \\
\hline No de nodos & 311,00 & 96,00 & 55,00 \\
\hline No de enlaces & 1439,00 & 696,00 & 410,00 \\
\hline Densidad & 0,02 & 0,15 & 0,27 \\
\hline $\begin{array}{l}\text { Grado mínimo } \\
\text { normalizado }\end{array}$ & 0,01 & 0,06 & 0,16 \\
\hline $\begin{array}{l}\text { Grado máximo } \\
\text { normalizado }\end{array}$ & 0,39 & 0,62 & 0,66 \\
\hline Grado promedio & 9,25 & 14,50 & 14,90 \\
\hline Diámetro & 5,00 & 4,00 & 3,00 \\
\hline $\begin{array}{l}\text { Centralización de } \\
\text { grado }\end{array}$ & 0,36 & 0,47 & 0,40 \\
\hline $\begin{array}{l}\text { Centralización de } \\
\text { la intermediación }\end{array}$ & 0,31 & 0,17 & 0,09 \\
\hline
\end{tabular}

Tabla 4. Indicadores de redes de coparticipación en tribunales de ByD. 1993-2006 el cluster $U G R$. El tamaño del nodo es proporcional al número de las presencias respectivas en tribunales mientras que el grosor del enlace es más intenso cuanto mayor haya sido el número de coincidencias entre dos actores, como se aprecia en las relaciones diádicas entre Caridad - Moreiro, Caridad - Sanz Casado o Sagredo - Ros García. El grosor expresa un mayor grado de similitud entre sí ya que comparten mayor número de atributos en común que, por ejemplo, lo que denotan los vínculos entre Abadal - Gómez Caridad o Asensi - Marzal.

La elevada proporción de miembros de las universidades Complutense, Carlos III, Granada y Murcia en detrimento de otras, que vemos en la figura 2, se parece a una red entre profesores que son conocidos entre sí y están más próximos. La mayor parte de los miembros de la red se interrelacionan con otros compañeros de la misma universidad y así sucede en los cinco subconjuntos en los que se ha descompuesto la red donde tan sólo uno de los nodos, Gómez - Caridad (Csic), no pertenece al sector universitario.

Es preciso observar las posiciones de los nodos, además de sus conexiones y atributos. Desde el punto de vista individual, la mayor presencia en tribunales ajenos a su propia universidad, le otorga al profesor Moreiro el mayor número de contactos diferentes con otros nodos de la red (36), junto con Sagredo (35) y J. López Yepes (34), e integran, junto con Caridad (27) y Sanz-Casado (27) el cluster de los miembros más "populares" ya que son los más frecuentemente elegidos por otros para formar parte de los tribunales. En el otro extremo se encuentran aquellos otros con menor grado de popularidad, como Moya (20) o RodríguezMuñoz (13), pese a su elevada producción como directores de tesis.

No son los únicos casos. En la figura 3 se muestra el resultado del cálculo de la correlación de Spearman entre los rangos de grado de los miembros de la red y los rangos del número de sus presencias en los tribunales.

La comparación entre ambos valores es significativa y muestra que en el conjunto hay una fuerte correlación entre ambas variables ya que el coeficiente de correlación es cercano a $1\left(\mathrm{R}^{2}=0,95\right)$. Esto se debe a que si se es miembro de muchos tribunales de tesis hay mayores posibilidades de entrar en relación con otros colegas que integran la red. Es muy informativo e interesante que haya un conjunto de profesores que pese a formar parte de muchos tribunales no incrementen su grado. Ello se debe a que pese a asistir a más tribunales no establecen un mayor número de vínculos "diferentes" con otros miembros "nuevos" de la red. Es una situación inversa a quienes, pese a coincidir con muchos profesores diferentes en los tri- 


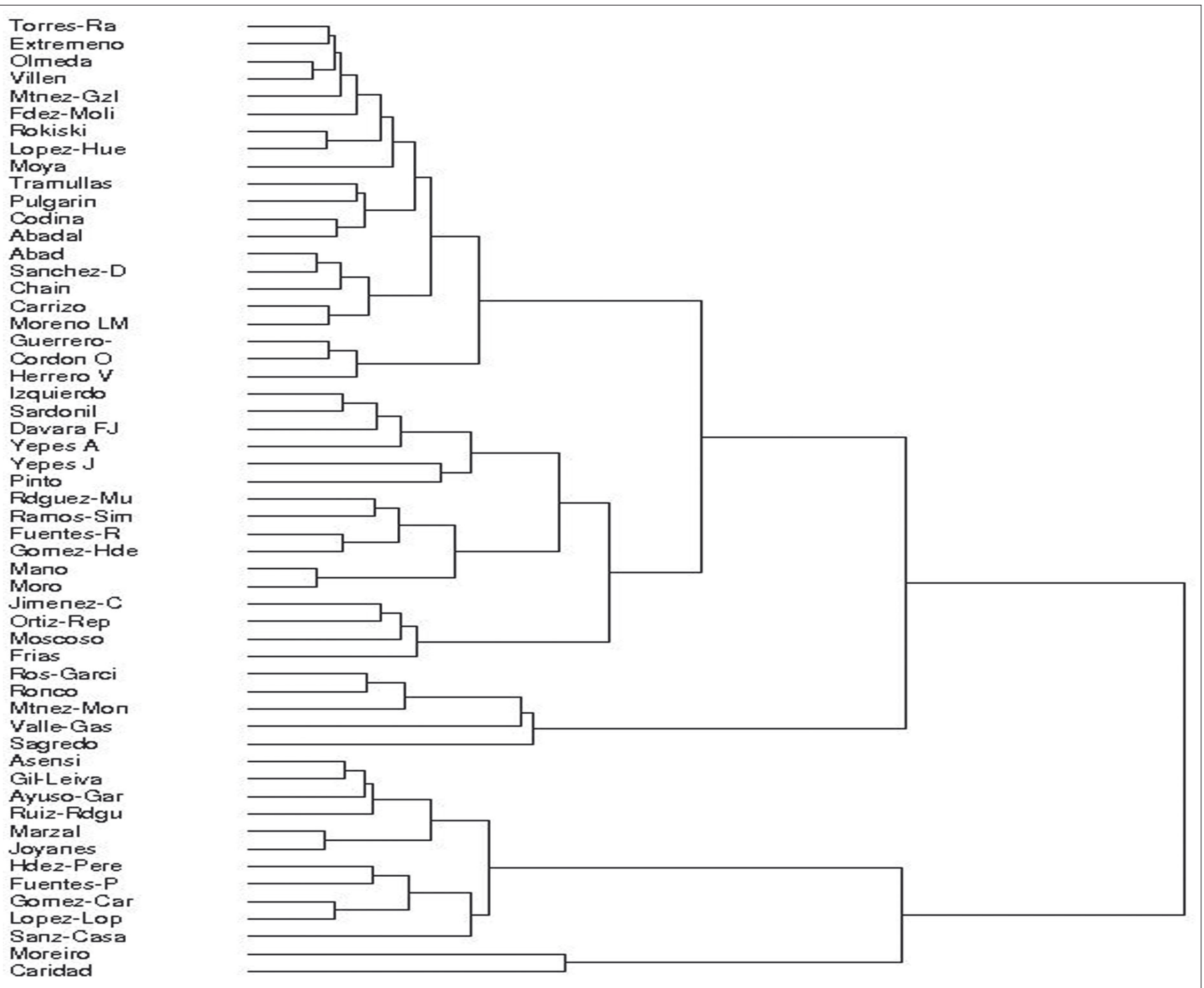

Figura 1. Estructura de clusters de la red de coparticipación en tribunales de ByD. Método de Ward. 1993-2006

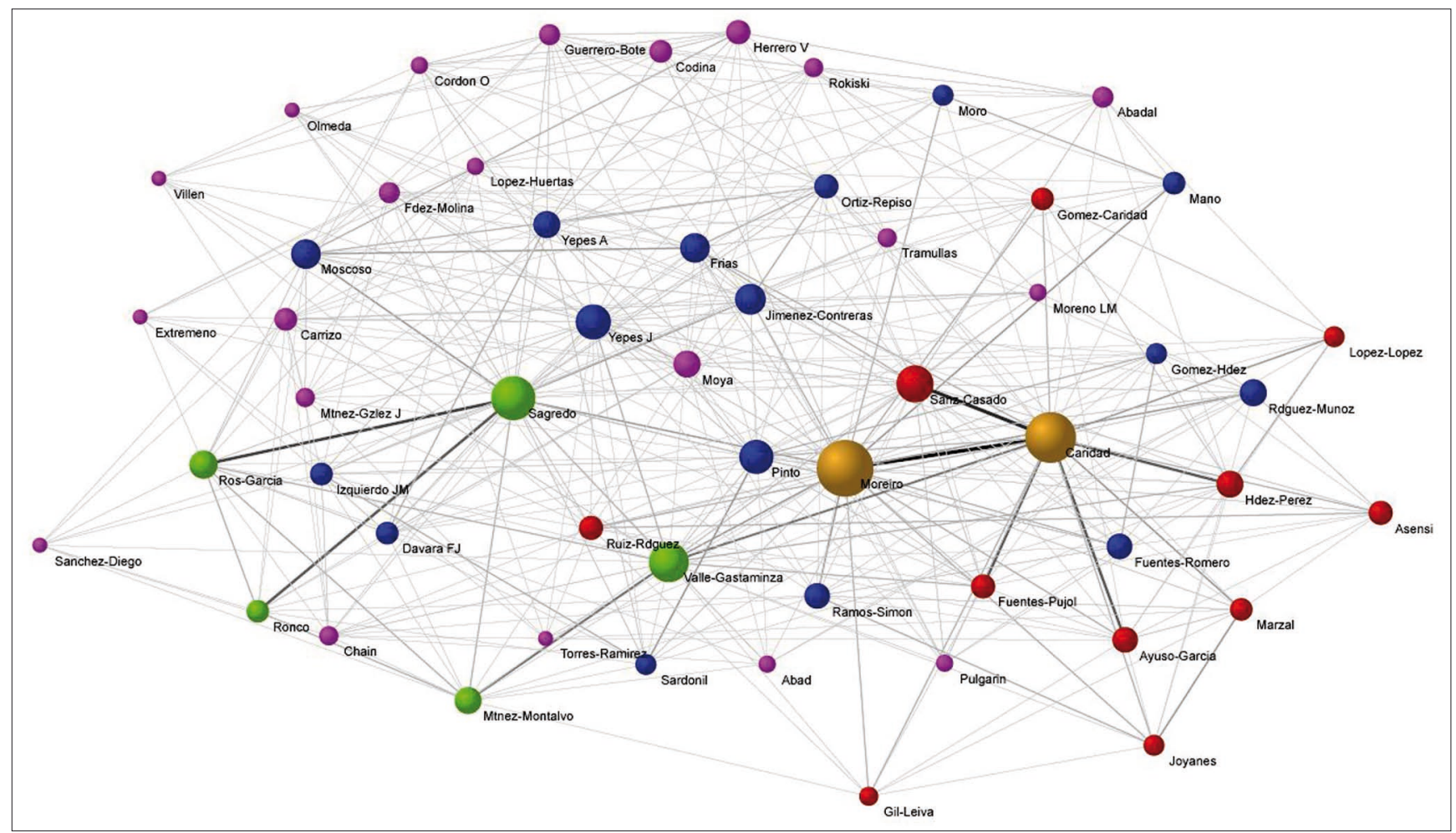

Figura 2. Red con 5 clusters 
bunales, ya que tienen mayor grado, son invitados o elegidos en menor medida a formar parte de tribunales de tesis. En el primer caso, parece que ese grupo de profesores coinciden con mayor frecuencia entre sí en diferentes tribunales con actores ya conocidos en tribunales previos, mientras que los segundos no.

Finalmente, hay que señalar que en conjunto, son los profesores catedráticos quienes ocupan el centro de la estructura de la red: López-Yepes J. y A., Sagredo, Moreiro, Caridad, Sanz-Casado, Pin-

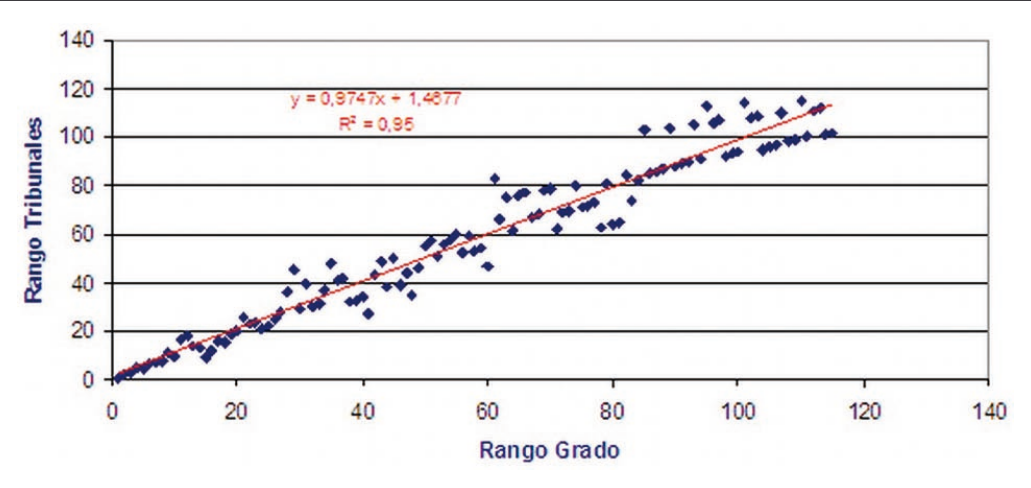

Figura 3. Rango de grado versus de asistencia a tribunales to, con las excepciones de Rokinski (UCM) e Izquierdo (Universidad de Murcia) y los profesores titulares, que ocupan la parte externa o periférica de la red.

\section{Discusión y conclusiones}

¿Qué mecanismos pueden explicar la configuración de esta red resultado de las coparticipaciones en tribunales de tesis doctorales de ByD en la universidad española?

El primer mecanismo que explica la articulación de una red formal de este tipo, se basa en el principio de la selección por otros para formar parte de los tribunales entre quienes se percibe que son más similares entre sí. Al seleccionar a quienes se piensa que son más similares, se reducen potenciales áreas de conflicto en la relaciones que se pueden derivar de la pertenencia a un tribunal cuya decisión implica un paso en la carrera del doctorado y del director de la tesis, al obtener ambos un incremento en el mérito en sus respectivas carreras profesionales.

\section{"Los análisis más informativos son los basados en múltiples relaciones"}

El mismo mecanismo se produce en el nivel diádico. Hay un conjunto de actores que se "eligen" entre sí los unos a los otros con mayor frecuencia. En esos subgrupos, no hay posibilidad que otros actores puedan "elegir" o "ser elegidos" para formar parte de los tribunales con los miembros de esos subgrupos ya que éstos constituyen un subconjunto cohesionado.

Un segundo mecanismo que explica el estilo de la vinculación hay que buscarlo en la proximidad física. No es infrecuente que los directores más activos, elijan para formar parte de tribunales a profesores de la misma universidad o de la misma provincia en la que coexistan diferentes universidades, ya que son los más próximos y han podido llevar a cabo con ellos una ma- yor frecuencia de contactos directos e informales que con otros colegas de otros departamentos o universidades. Al mismo tiempo, la asistencia a esos actos ofrece a los directores la oportunidad de explorar la extensión en la que demuestran el grado coincidencia o discrepancia y hasta qué punto les pueden inspirar confianza para ser convocados en actos futuros.

Los más coincidentes con otros miembros en la red, los integrantes de esos subconjuntos cohesionados por la intensidad de sus vínculos, lo son por las similitudes existentes entre ellos, que les proporcionan individualmente una base para legitimar su propia identidad social como integrantes de una determinada categoría. Este mecanismo se retroalimenta y se repite en futuros actos académicos de la misma naturaleza a lo largo del tiempo.

Desde el punto de vista nodal, aquellos actores con mayor número de relaciones diferentes pueden verse influidos en la percepción de la red ya que gracias a esa posición más central pueden tener una percepción más exacta de la estructura social que subyace en la disciplina. Esto sucede porque están mejor conectados directa e indirectamente con otros colegas diferentes en la red y así adquieren una "vista de pájaro" capaz de ver toda la red al completo. Los actores situados en la periferia, tienen una visión menos aguda para percibir en la red de la disciplina "quién sabe qué" y "quién conoce a quien sabe qué".

¿Qué clases de actores en la red son más equivalentes? Según el sistema de relaciones, los que exhiben un patrón más similar de enlace. En nuestro caso, los actores integrados en los cinco clusters que tienen un patrón similar de enlace. De acuerdo con el análisis efectuado, puede afirmarse que los nodos integrantes en cada clase o cluster ocupan posiciones sociales similares en la red, lo que no implica que sean subgrupos cohesionados.

Basándonos en los datos de coparticipación en tribunales de tesis doctorales, se han identificado las clases de profesores en la ByD española que ocupan las mismas posiciones en la red social, derivadas del 
conjunto de vínculos de una sola relación. Los análisis más informativos son los basados en múltiples relaciones. La base de datos de este trabajo es débil y debe mejorarse significativamente. Nuestro análisis es limitado, pero las clases identificadas son coherentes según el tipo de relación analizada y abre perspectivas para abordar el análisis de redes de relaciones múltiples en el mismo dominio.

\section{Bibliografía}

Barjak, F. "The role of the internet in informal scholarly communication". Journal of the American Society for Information Science and Technology, 2006, v. 57, n. 10, pp. 1350-1367.

Batagelj, V.; Ferligoj, A.; Doreian, P. "Direct and indirect methods for structural equivalence". Social networks, 1992, v. 14, n. 1-2, pp. 63-90

Batagelj, V.; Zaversnik, M. "An O(m) algorithm for cores decomposition of networks". 2002. Consultado en 15-07-2008.

http://arXiv:cs/0310049v1

Brieger, R. L. "Career attributes and network structure: a blockmodel study of a biomedical research specialty". American sociological review, 1976, v. 41, n.1, pp. 117-135.

Crane, D. Invisible colleges: diffusion of knowledge in scientific communities. Chicago: University Chicago Press, 1975.

Delgado-López-Cózar, E.; Torres-Salinas, D.; Jiménez Contreras, E. Ruiz-Pérez, R. "Análisis bibliométrico y de redes sociales aplicado a las tesis bibliométricas defendidas en España (1976-2002): temas, escuelas científicas y redes académicas". Revista española de documentación científica, 2006, v. 29, n. 4, pp. 493-524.

Freeman, L. C. "The impact of computer based communication on the social structure of an emerging scientific specialty". Social networks 1984, v 6, n. 3, pp. 201-221.

Fuentes-Pujol, M. E.; González-Quesada, A. "Tesis doctorales en España en información y documentación, 1976-2002”. En: Morán, M. A.; Rodríguez-López, M. C. La documentación para la investigación. Homenaje a José Antonio Martín Fuertes. León: Universidad de León, 2002, pp. 229 242.

Garvey W. D.; Griffith, B. C. "Informal channels of communication in the behavioral sciences: their relevance in the structuring of formal or bibliographic communication". En: Montgomery, E. B. (ed.). The foundation of access to knowledge. Syracuse: Syracuse University, 1967, pp. 129-146.

López-Yepes, J. "Investigadores y directores de tesis doctorales, creadores de focos de investigación y de escuelas científicas en documentación. E caso del Departamento de Biblioteconomía y Documentación de la Universidad Complutense de Madrid". En: ISKO. V. La representación y organización del conocimiento: Metodologías, modelos y aplicaciones. Alcalá de Henares: Universidad de Alcalá de Henares, 2001, pp. 443-477.

López-Yepes, J. "Focos de investigación y escuelas científicas en documentación. La experiencia de las tesis doctorales". El profesional de la información, 2002, v. 11, n. 1. pp. 46-52.

López-Yepes, J.; Fernández-Bajón, M. T.; Prat-Sedeño, J. "Las tesis doctorales en biblioteconomía y documentación. Diagnóstico y propuesta de criterios de evaluación". Documentación de las ciencias de la información, 2005, v. 28, pp. 173-187.

Moya-Anegón, F. de; Vargas-Quesada, B.; Chinchilla-Rodríguez, Z; Corera-Álvarez, E.; González-Molina, A.; Muñoz-Fernández, F. J.; Herrero-Solana, V. "Visualización y análisis de la estructura científica española: ISI Web of science 1990-2005“. El profesional de la información, 2006, v. 15, n. 4, pp. 258-269.

Newman, M. E. J. "Coauthorship networks and patterns of scientific collaboration". Proceedings of the National Academy of Sciences of the United States of America, 2004, n. 101 (suppl. 1), pp. 5200-5205.

Orera-Orera, L. "Las tesis sobre biblioteconomía y bibliotecas y su accesibilidad a través de las bibliotecas universitarias españolas”. En: López
Yepes, J.; López, P.; Fernández Bajón, Ma T. (coord.). Estudios de biblioteconomía y documentación: homenaje a la profesora María Rosa Garrido Arilla. Madrid: Universidad Complutense, 2004, pp. 91-102.

Perianes-Rodríguez, A. Análisis de las redes sociales de los tribunales de tesis en dos departamentos españoles de biblioteconomía y documentación. Universidades de Granada y Carlos III de Madrid. Madrid: Universidad Carlos III, septiembre 2004 [Manuscrito no publicado].

Price, D. J. de Solla; Beaver, B. de. "Collaboration in an invisible college". American psychologist, 1966, v. 21, n. 11, pp.1011-1017.

Price, D. J. de Solla. "Ciencia y tecnología: distinciones e interrelaciones". En: Barnes, B. (comp.). Estudios sobre sociología de la ciencia. Madrid: Alianza, 1980, pp. 163-177.

Scott, J. Social network analysis: a handbook. Londres: Sage, 1992.

Sierra, G. "Deconstrucción de los tribunales del Csic en el período 19852002: profesores de investigación en el área de física". Apuntes de ciencia y tecnología, 2003, n. 7, pp. 30-40.

Small, H. "Cocitation context analysis and the structure of paradigms". Journal of documentation, 1980, v. 36, n. 3, pp. 183-196.

Small, H. G.; Crane, D. "Specialties and disciplines in science and social science: an examination of their structure using citation indexes". Scientometrics, 1979, v. 1, n. 5-6, pp. 445-461.

Valle-Gastaminza, F. del. "Trayectoria personal del Departamento de Biblioteconomía y Documentación en la Facultad de Ciencias de la Información de la Universidad Complutense de Madrid". Documentación de las ciencias de la información, 2002, v. 25, pp. 87-93.

Vargas-Quesada B.; Moya-Anegón F. de.; Chinchilla-Rodríguez, Z.; Corera-Álvarez, E.; Guerrero-Bote, Vicente-P. "Evolución de la estructura científica española: ISI Web of science 1990-2005”. El profesional de la información, 2008, v. 17, n.1, pp. 22-37.

Wasserman S.; Faust, K. Social network analysis: methods and applicatio$n s$. Cambridge: Cambridge University Press, 1994.

White, H. D.; McCain, K. W. "Visualizing a discipline: an author co-citation analysis of information science, 1972-1995". Journal of the American Society for Information Science, 1998, v. 49, n. 4, pp. 327-355.

White, H. D.; Wellman, B.; Nazer, N. "Does citation reflect social structure? Longitudinal evidence from the "Globenet" interdisciplinary research group". Journal of the American Society for Information Science and Technology, 2004, v. 55, n. 2, pp. 111-126.

Zapico-Alonso, F.; Barragán, M. R.; Guerrero-Bote, Vicente-P.; LópezPujalte, C. "La investigación en documentación: análisis bibliométrico de las tesis en documentación”. En: Morán, M. A.; Rodríguez-López, M. C. La documentación para la investigación. Homenaje a José Antonio Martín Fuertes. León: Universidad de León, 2002, pp. 689-713.

Ziman, J. ¿Qué es la ciencia? Madrid: Cambridge University Press, 2003.

\section{Carlos Olmeda-Gómez, Antonio Perianes-Rodríguez} y $M^{a}$ Antonia Ovalle-Perandones, Departamento de Biblioteconomía y Documentación. Universidad Carlos III de Madrid,

C/Madrid 128, 28903 Getafe (Madrid), España.

carlos.olmeda@uc3m.es

antonio.perianes@uc3m.es

movalle@bib.uc3m.es

\section{Félix Moya-Anegón}

Centro de Ciencias Humanas y Sociales. Consejo Superior de Investigaciones Científicas,

Albasanz 26-28, 28037 Madrid, España.

felix@ugr.es 